\section{P371 TWO MISSED DIAGNOSED PATIENTS WITH STING- ASSOCIATED VASCULOPATHY WITH ONSET IN INFANCY IN CHINA}

Liping Jiang*, Yao Cao. Children's Hospital of Chongqing Medical University, Chongqing, China

\subsection{6/archdischild-2019-epa.717}

Background and aims Stimulator of interferon genes (STING)associated vasculopathy with onset in infancy (SAVI) is firstly described in 2014 as a type I interferonopathy that resulting from heterozygous mutations of TMEM173. This gene encodes the STING adaptor protein, and mutations lead to a gain of function on STING and overproduction of interferon beta. SAVI is characterized by neonatal-onset systemic inflammation, a severe cutaneous vasculopathy and interstitial lung disease. JAK inhibitors are considered as an effective therapeutic strategy. We sought to describe two missed diagnosed patients with SAVI (P1 and $P 2$ ) in order to draw attention to this illness.

Methods The clinical data were collected and Sanger sequencing of the gene TMEM173 was performed. An introspection of missed diagnosis and differential diagnosis was discussed.

Results The two boys shared similar manifestations including recurrent skin abscess in winter with skin lesions and recurrent respiratory tract infections since their births. It occurred to us neutrophil defects like CGD but NBT test and DHR123 were normal for both of them in 2011. Peroxidase staining was positive and no mutations in MYD88 (P1). Computed tomography of the chest revealed pulmonary fibrosis yet no relevant genes (including $A B C A 3, S F T P C$ ) mutations were found. Joint pain was significant for $P 2$ but Naproxen was ineffective. Treatments with antibiotics turned out little improvement and wouldn't prevent the progression. Finally, both of them died of respiratory and circulatory failure in 2012 and 2016 respectively. Recently, genetic mutations (c.463 $\mathrm{G}>\mathrm{A}$ and c.461 $\mathrm{A}>\mathrm{G}$ ) in exon5 of TMEM173 were discovered, confirming the diagnosis of SAVI.

Conclusions Owing to unfamiliarity with this disease, some cases would have been misdiagnosed or missed diagnosed. Thus, we propose that SAVI should be taken into consideration for children with chilblain skin lesions and pulmonary fibrosis after ruling out other related genes mutations.

\section{P372 CLINICAL CHARACTERISTICS OF A CHILD WITH MENDELIAN SUSCEPTIBILITY TO MYCOBACTERIAL DISEASE DUE TO MUTATIONS OF IL12RB1}

Liping Jiang*. Children's Hospital of Chongqing Medical University , Chongqing, China. Children's Hospital of Chongqing Medical University, Chongqing, China

\subsection{6/archdischild-2019-epa.718}

Background and aims Autosomal recessive interleukin-12 receptor $\beta 1$ (IL-12R $\beta 1$ ) deficiency is the most common cause of Mendelian susceptibility to mycobacterial disease (MSMD). Here we report a case with multi-drug resistant tuberculosis (MDR-TB) due to mutations of IL12RB1 to investigate the clinical characteristics of MSMD.

Methods The clinical features of a child with mutations of IL12RB1 were summarized and the mutations were analyzed by Sanger sequencing.
Results The 10-year-old boy was vaccinated with Bacille Calmette-Guérin (BCG) at birth, and suffered BCG disease within 3 months of age. A progressive left side axillary adenopathy was developed, then infections disseminated to the lung, thoracic cavity, peritoneal cavity, intestine, brain, skin, and ear. The patient was diagnosed with MSMD due to IL12RB1 deficiency at five years old. There were no significant abnormalities in routine immunological examinations including lymphocyte subsets, immunoglobulins, complement and neutrophil respiratory burst test. The child didn't receive early and standard anti-tuberculosis therapies after onset, and the disease progressed to MDR-TB. During the whole disease course, the anti-tuberculosis treatments were adjusted several times owing to poor responses. rIFN- $\gamma$ was added twice a week but stopped because of the adverse reaction of fever. The infections were uncontrolled. Genetic testing revealed the compound heterozygous IL12RB1 mutations c.632G $>\mathrm{C}$ in exon 7 and c.1106T $>\mathrm{C}$ in exon 10 inherited from farther and mother respectively. Those variations lead to $\mathrm{R} 211 \mathrm{P}$ and I369T amino acid changes reported previously.

Conclusions Mutations of IL12RB1 can lead to severe MSMD. When there were no obvious abnormalities in routine immunization assessments of patients with BCG disease, the possibility of MSMD needs to be considered. IL12RB1 protein detection and gene analysis are helpful for diagnosis. Standard antituberculosis treatments are conducive to tuberculosis or BCG infection control. The efficacy should be evaluated to determine whether to stop it when adverse reactions of $\mathrm{rIFN}-\gamma$ happen.

\section{P373 YOU GIVE ME FEVER! - THE AUTOINFLAMMATORY CLINIC IN AN IRISH TERTIARY PAEDIATRIC HOSPITAL}

1Jayne MacMahon*, ${ }^{1}$ Ana Louise Hawke, ${ }^{1}$ Emma J MacDermott, ${ }^{2}$ Patrick Gavin, ${ }^{2}$ Karina Butler, ${ }^{2}$ Timothy Ronan Leahy, ${ }^{1}$ Orla G Killeen. ${ }^{1}$ National Centre for Paediatric Rheumatology, Our Lady's Children's Hospital Crumlin, Dublin, Ireland; 'Department of Infectious Diseases, Our Lady's Children's Hospital Crumlin, Dublin, Ireland

\subsection{6/archdischild-2019-epa.719}

Introduction Auto-inflammatory syndromes (AIS) are inherited disorders of the innate immune system that lead to pathogenic inflammation. Multiple organ systems can be involved and disease can lead to significant end organ damage. Early diagnosis and treatment may improve outcome; ${ }^{1}$ Diagnosis is often delayed due to the low incidence of these disorders. An autoinflammatory clinic was established in OLCH Crumlin, Dublin, a tertiary level Paediatric Hospital, in November 2015 to assess patients with known or suspected auto-inflammatory disease within a multi-disciplinary setting. This clinic is attended by immunology, rheumatology and dermatology specialists with multi-disciplinary team input as required.

Aim The aim of this study was to survey the diagnosis and management of patients attending this clinic over a 2 and a half year period.

Methods A retrospective observational chart review of all patients attending the Autoinflammatory clinic from November 2015 to June 2018. Age of onset, demographic details, diagnosis (if known) and management were documented. Details of any genetic analysis if undertaken were also included.

Results A total of 47 patients attended the auto-inflammatory clinic over the identified period. Age of onset at first 
symptoms ranged from birth to 15 years. A diagnosis of an AIS was made in 57\% with PFAPA (periodic fever, aphthous stomatitis, pharyngitis, adenitis) accounting for the majority. An undetermined autoinflammatory syndromes was suspected in $40 \%$. 3\% who attended had no symptoms or features supportive of an AIS. Overall, 36\% were found to have a confirmed genetic mutation, either benign or pathogenic and 28\% had a positive family history of auto-immune or auto-inflammatory syndrome in a $1^{\text {st }}$ degree relative. Colchicine monotherapy $(32 \%)$ was the first choice of therapy followed by anakinra monotherapy (19\%) or a combination of both. All treated patients reported symptom improvement on therapy with the exception of 2 .

Conclusion This study gives an overview on the spectrum of autoinflammatory disease presenting in Ireland and their current management. The majority of patients attending were appropriately referred to this clinic and had improvement in symptoms following initiation of treatment.

\section{REFERENCE}

1. Ter Haar NM, Oswald M, Jeyaratnam J, et al. Recommendations for the management of autoinflammatory diseases. Ann Rheum Dis 2015;74:1636-1644.

\section{P374 LARYNGEAL SARCOIDOSIS RESPONSIVE TO TREATMENT WITH SIROLIMUS: A FIRST CASE REPORT}

${ }^{1}$ Karen Kelleher*, ${ }^{2}$ John Russell, ${ }^{3}$ Orla Killeen, ${ }^{3}$ Emma Jane MacDermott, ${ }^{1}$ Timothy Ronan Leahy. 'Department of Paediatric Immunology, OLCH, Crumlin, Dublin, Ireland; ${ }^{2}$ Department of Paediatric Otorhinolaryngology, OLCH, Crumlin, Dublin, Ireland; ${ }^{3}$ Department of Paediatric Rheumatology, OLCH, Crumlin, Dublin, Ireland

10.1136/archdischild-2019-epa.720

We report the case of a 15 year old girl with a four month history of gradual onset dysphonia and dysphagia. Endoscopy revealed a significantly enlarged epiglottis and arytenoids causing supraglottic airway obstruction (pinpoint airway). She progressed to emergency tracheostomy and a biopsy of the epiglottis was taken. Histology revealed lymphoid hyperplasia with focal non-necrotizing granulomata. A screen for infection was negative, as was a serum ACE. The patient was commenced on corticosteroids and methotrexate, but after three months, there was no improvement. Therafter, she was switched to sirolimus, which led to resolution of her epiglottic swelling, allowing the tracheostomy to be reversed and corticosteroid therapy to be discontinued.

Conclusion This is the first case, to our knowledge, of sirolimus being successfully used in treatment of laryngeal sarcoidosis. Sirolimus could be considered a treatment option in these instances, particularly in the presence of lymphoid aggregates on histology.

\section{P375 QUALITY OF LIFE ASSESSMENT IN PATIENTS WHO RECEIVES SCIG AND IVIG}

Sukru Cekic, Yasin Karali, Fatih Cicek, Sara Sebnem Kilic*. Uludag University Faculty of Medicine, Pediatric Allergy and Clinical Immunology , Bursa, Turkey

\subsection{6/archdischild-2019-epa.721}

Introduction Primary immunodeficiency diseases (PIDs) describe a group of clinically and genetically heterogeneous disorders that afflict lymphoid and myeloid lineages.
Immunoglobulin replacement therapy has been the standard treatment for patients with antibody production deficiency since the 1950s. Human serum immunoglobulin manufactured from pools of donated plasma can be administered intramuscularly, intravenously or subcutaneously. Subcutaneous route is a safe and efficacious method to prevent serious bacterial infections in patients experiencing difficulties with IVIG.

Aim Treatment satisfaction and HRQOL in patients with PIDs was evaluated upon switching from intravenous (IVIG) or subcutaneous immunoglobulins (SCIGs) to $10 \%$ SCIG (Kiovig ${ }^{\circledR}$ ).

Material and methods Twelve patients who received SCIG and 14 patients who received IVIG were included in the study. THE WHOQOL-BREF questionnaire was applied to all cases. The WHOQOL-BREF https://www.who.int/substance_abuse/ research_tools/en/english_whoqol.pdf.

Results Male to female ratio was $8 / 4$ in SCIG group and 8/6 in intravenous IgG (IVIG) group. The median age was 31.2 years (minimum $=5$ years, maximum $=49$ years) in SCIG group and 23.2 years (minimum $=5$ years, maximum $=47$ years) in IVIG group. All patients who received SCIG were taking IVIG before. When the median scores of the responses to each question were examined according to before and after SCIG treatment; improvement was seen in 13 questions, deterioration in 2 questions. When the total quality of life score was evaluated before and after SCIG and in the IVIG group, the highest median score belonged to the patients receiving SCIG (median $=104$, minimum $=44$, maximum $=130$ ). In addition, the total quality of life score was found to be 10 points higher in SCIG patients than before. The questions with deterioration in the answers were 'How much do you need any medical treatment to function in your daily life?' and 'Are you able to accept your bodily appearance?'. We think that the worsening of these questions can be due to recurrent injections and local side effects. No serious side effects were seen in both groups. SCIG was performed without any problem in a female pregnant patient who had anaphylactoid reactions to different IVIG brands.

Conclusion The number of studies which investigated the effects of the SCIG treatment on quality of life in Turkey were limited. In this study, SCIG was found to be a safe treatment modality that improves the quality of life.

\section{P376 PARECHOVIRUS INFECTION AS THE PRESENTING ILLNESS IN AN INFANT WITH SCID}

${ }^{1}$ Elizabeth Murphy*, ${ }^{1}$ Gearoid McGauran, ${ }^{1}$ David Coghlan, ${ }^{2}$ Ronan Leahy, ${ }^{1}$ Claire Purcell. 'Department of General Paediatrics, Tallaght University Hospital, Dublin, Ireland; ${ }^{2}$ Department of Paediatric Immunology, Our Lady's Children's Hospital Crumlin, Dublin, Ireland

\subsection{6/archdischild-2019-epa.722}

Introduction Severe Combined Immunodeficiency (SCID) is a heterogenous group of primary immunodeficiency disorders with impaired cellular and humoral immunity. This leads to increased susceptibility to life-threatening bacterial, viral and fungal infections. Incidence is approximately 1 per 58,000 live births. ${ }^{1}$ Typically, SCID presents in the first months of life with severe, recurrent infections, failure to thrive, dermatologic manifestations or chronic diarrhoea. We present a case of newly diagnosed SCID and examine parechovirus viral loads before and after treatment. 